\title{
A Modified Adaptive Sparse Channel Estimator for OFDM Systems Based On Singular Value Decomposition
}

\author{
Liu Zhiyong $^{1}$, Wang Yirong $^{2}$ and Liu Weicai ${ }^{3}$ \\ ${ }^{1,2}$ Beijing GuoDianTong Network Technology Co., Ltd. \\ ${ }^{3}$ School of Electronic Engineering, Beijing University of Posts and \\ Telecommunications \\ ${ }^{1}$ liuzhiyong@sgitg.sgcc.com.cn, ${ }^{2}$ wangyirong@sgitg.sgcc.com.cn, \\ 3liuweicai2014@163.com
}

\begin{abstract}
In this paper, a modified adaptive sparse channel estimator based on singular value decomposition (SVD) for OFDM systems is proposed. The conventional adaptive sparsity matching pursuit (ASMP) based compressive channel estimation has bad anti-noise performance, although not needing the information of sparsity. Because using the SVD to modify the measurement matrix of CS can improve the robustness to noise. So we use the SVD to modify the measurement matrix of ASMP based compressive channel estimation. The proposed channel estimation has better robustness to noise and low error. The simulation results show that comparing with ASMP based compressive channel estimation, the proposed algorithm has $1 \mathrm{~dB}$ gain at MSE and $1.3 \mathrm{~dB}$ gain at BER.
\end{abstract}

Keywords: channel estimation, compressive sensing, adaptive sparsity matching pursuit, Singular Value Decomposition, OFDM.

\section{Introduction}

The Orthogonal frequency division multiplexing (OFDM) has been widely applied in wireless communication systems because of its high spectrum efficiency and characteristic of multi-path resistance. In order to achieve a better performance, the OFDM systems typically adopt the coherent detection. In the coherent detection, the channel state information (CSI) is crucial and can be obtained by using pilot-aid channel estimation. The traditional pilot aided channel estimations[1]-[3] are nonparametric. In order to achieve precise CSI, numbers of pilots need to be inserted which turns to lower the system resource utilization.

Recently, the compressed sensing (CS) has been applied into the channel estimations by exploiting the sparsity of wireless channel. And the CS based channel estimation can achieve better performance than traditional method with less number of pilots. In the CS based channel estimation, the methods with greedy reconstruction algorithms are widely studied because of its low complexity. The typical greedy reconstruction algorithms include orthogonal matching pursuit (OMP)[4], regularized orthogonal matching pursuit (ROMP)[5] and CoSaMP[6]. However, these methods require the sparsity of channel which is hard to achieve in practical applications. Some modified CS based channel estimations [7-10] were proposed to solve the problem of the unknown channel sparsity. Especially, an adaptive sparse channel estimation[10] was proposed which employs the adaptive sparsity matching pursuit (ASMP)[11] reconstruction algorithm.

However, the anti-noise performance of ASMP based channel estimation is not satisfactory. In [12], one singular value decomposition (SVD) noise reconstruction algorithm was presented to improve the performance of anti-noise of CS. In this paper, we adopt the method proposed in [12] to modify the observation matrix of ASMP based channel estimator. 


\section{System Model}

We consider the number of total carriers is $N$. The total number of pilot subcarriers is $N_{p}$ with positions represented by $k_{1}, k_{2}, k_{3} \cdots, k_{N_{p}}\left(1 \leq k_{1}<k_{2}<k_{3} \cdots<k_{N_{p}} \leq N\right)$.

In matrix notation, the received pilot vector $Y$ is given by

$$
Y_{p}=X_{p} F h+W=A h+W
$$

where $X_{p}$ is a diagonal matrix with pilot symbol on its diagonal; $h=[h[0], h[1], \ldots, h[L-1]]^{T}$ is the CIR vector; $F$ is sub-matrix selected by the row indices $\left[k_{1}, k_{2}, k_{3} \cdots, k_{N_{p}}\right]$ and column indices $[0,1, \cdots, \mathrm{L}-1]$ from the standard $N \times N$ Fourier matrix; $W=\left[\mathrm{w}(0), \mathrm{w}(1), \cdots, \mathrm{w}\left(N_{p}-1\right)\right]^{T}$ is the noise term.

Because the system sampling interval is much smaller compared to channel delay spread, $h$ is a sparse vector which means that most coefficients are zero or nearly zero. The conventional nonparametric channel estimation requires the number of pilots $N_{p}$ larger than the channel length $L$. But in CS based channel estimator, $N_{p}$ is smaller than the channel length $L$ which means that the number of pilot is less. We assume that the number of nonzero element of $h$ is $S$ and $S \square L$.So we can achieve $h$ from (1) by solving the following 10-norm minimization problem

$$
\min _{h}\|h\|_{0} \text { s.t. }\left\|Y_{p}-A h\right\|_{2} \leq \delta
$$

Unfortunately, solving formula (2) is an NP-Hard problem. However, the 10-norm minimization is transformative to 11-norm minimization

$$
\min _{h}\|h\|_{1} \text { s.t. }\left\|Y_{p}-A h\right\|_{2} \leq \delta
$$

To solve the convex program, we often used reconstruction algorithm which is a very important in the CS. Reconstruction algorithms include greedy algorithm, convex relaxation methods and combination algorithm. In this paper, we mainly study the Greedy algorithm based channel estimators.

\section{ASMP Based Channel Estimator}

ASMP greedy reconstruction algorithm does not require the sparity of signal and takes advantage of backtracking to refine the chosen supports and the current approximation in the process. In [10], the ASMP based channel estimator was proposed. The ASMP based channel estimator is as follow.

Input: the received pilot vector $Y_{p}$, the observation matrix $A$;

Initialization: the residual $r=Y_{P}, \hat{h}=0$;

While (stopping criterion false) \{Outer loop

(1) $v=A^{T} r$

(2) $\Omega=\left\{j:|v(j)| \geqslant \tau\|r\|_{2}\right\}$

\{Form signal proxy

\{Thresholding

$$
P=\|\Omega \cup \operatorname{supp}(\hat{h})\|_{0}
$$

$\{$ Sparsity estimation $\}$

$j=1 ; \quad r_{0}=r ; h_{0}=\hat{h}$

\{Initialization of the inner loop $\}$

For $j:=1,2, \ldots, d o$

(a) $\quad u=A^{T} r_{j-1}, \quad \Lambda=\operatorname{supp}(u \mid P)$

$\{$ Identify supports $\}$ 
(b) $\quad \Gamma=\Lambda \cup \operatorname{supp}\left(h_{j-1}\right)$

\{Merge supports\}

(c) $\quad x=\arg \min _{h^{\prime} \text { supp }\left(h^{\prime}=\Gamma\right)}\left\|A h^{\prime}-Y_{p}\right\|$

\{LS estimation $\}$

(d) $\quad h_{j}=\left.x\right|_{P}$

\{Prune the estimate\}

(e) $\quad r_{j}=Y_{p}-A h_{j}$

\{Update error residual\}

(f) If $\left\|r_{j}\right\|_{2} \geqslant\left\|r_{j-1}\right\|_{2}$, then

\{stage switching

$r=r_{j-1}$;

$\hat{\mathrm{h}}=\mathrm{h}_{j-1}$;

go to step (1);

(g) $j=j+1$

\section{End For}

\section{End While}

$$
\text { loop\} }
$$

return $\hat{\mathrm{h}}=\mathrm{h}_{j}$

loop\}

\section{The Modified Method Based on Singular Value Decomposition}

Because the ASMP based channel estimation adopt original observation matrix, the robustness to noise is not satisfactory. In [12], a reconstruction algorithm was proposed using SVD to improve the reconstruction accuracy for noise signal. So, we reference this method and propose the modified channel estimation based on Singular Value Decomposition. The proposed method is as follow:

1. achieve the new observation matrix

(1) decompose the original observation matrix $A$ with SVD: $A=U\left(\begin{array}{ll}\Delta & 0 \\ 0 & 0\end{array}\right) V^{H}$; where $\Delta=\operatorname{diag}\left(\sigma_{1}, \sigma_{2}, \cdots, \sigma_{N_{p}}\right)$ is a diagonal matrix; $U$ and $V$ are unitary matrix; $V^{H}$ is complex conjugation of $V$;

(2) calculate the mean of elements of $\Delta$ : Mean $=\left(\sigma_{1}+\sigma_{2}+\cdots+\sigma_{N_{p}}\right) / N_{p}$

(3) achieve the new diagonal matrix: $\bar{\Delta}=\operatorname{diag}\left(\sigma_{1}^{\prime}, \sigma_{2}^{\prime}, \cdots, \sigma_{N_{p}}^{\prime}\right)$, where

$\sigma_{1}^{\prime}=\sigma_{2}^{\prime}=\cdots=\sigma_{N_{p}}^{\prime}=$ Mean

(4) achieve the new observation matrix: $\bar{A}=U\left(\begin{array}{cc}\bar{\Delta} & 0 \\ 0 & 0\end{array}\right) V^{H}$

2. ASMP based channel estimation with new observation matrix $\bar{A}$ Input: the received pilot vector $Y_{P}$, the new observation matrix $\bar{A}$; Initialization: the residual $r=Y_{P}, \hat{h}=0$;

While (stopping criterion false) \{Outer loop

(1) $\quad v=\bar{A}^{T} r$

(2) $\Omega=\left\{j:|v(j)| \geqslant \tau\|r\|_{2}\right\}$

\{Form signal proxy\}

(3) $\quad P=\|\Omega \cup \operatorname{supp}(\hat{h})\|_{0}$

\{Thresholding

$j=1 ; r_{0}=r ; h_{0}=\hat{h}$

\{Sparsity estimation\}

For $\mathrm{j}=1,2, \ldots$, do

(a) $\quad u=\bar{A}^{T} r_{j-1}, \Lambda=\operatorname{supp}(u \mid P)$

\{Identify supports $\}$

(b) $\quad \Gamma=\Lambda \cup \operatorname{supp}\left(h_{j-1}\right)$

\{Merge supports\}

(c) $\quad x=\arg \min _{h^{\prime}: \sup \left(h^{\prime}=\Gamma\right)}\left\|\bar{A} h^{\prime}-Y_{p}\right\|$

\{LS estimation $\}$ 
(d) $\quad h_{j}=\left.x\right|_{P}$

(e) $\quad r_{j}=Y_{p}-\bar{A} h_{j}$

(f) If $\left\|r_{j}\right\|_{2} \geqslant\left\|r_{j-1}\right\|_{2}$, then

$r=r_{j-1}$;

$\hat{\mathrm{h}}=\mathrm{h}_{j-1}$;

go to step (1)

(g) $j=j+1$

End For

loop

End While

loop

return $\hat{\mathrm{h}}=\mathrm{h}_{j}$

\section{Simulation Results and Analysis}

In this section, we will verify the performance of the proposed channel estimation by simulations and the steps are already mentioned above. We choose the shortwave multipath fading channel with four paths to make simulations. The QPSKOFDM system parameters: $N=1024, N_{p}=36, L=40$. And the pilot pattern is random pilot.

On account of the differences with different pilots arrangements, we should simulated the three channel estimators. Figure 1 shows the mean-square-errors (MSEs) of three channel estimators. Firstly, the conventional OMP based channel estimator has a poor performance. Secondly, the ASMP based channel estimator is better than OMP based method. Thirdly, the proposed method outperforms the other two channel estimators in overall SNRs. The proposed method has about $1 \mathrm{~dB}$ gain comparing with ASMP based channel estimator.

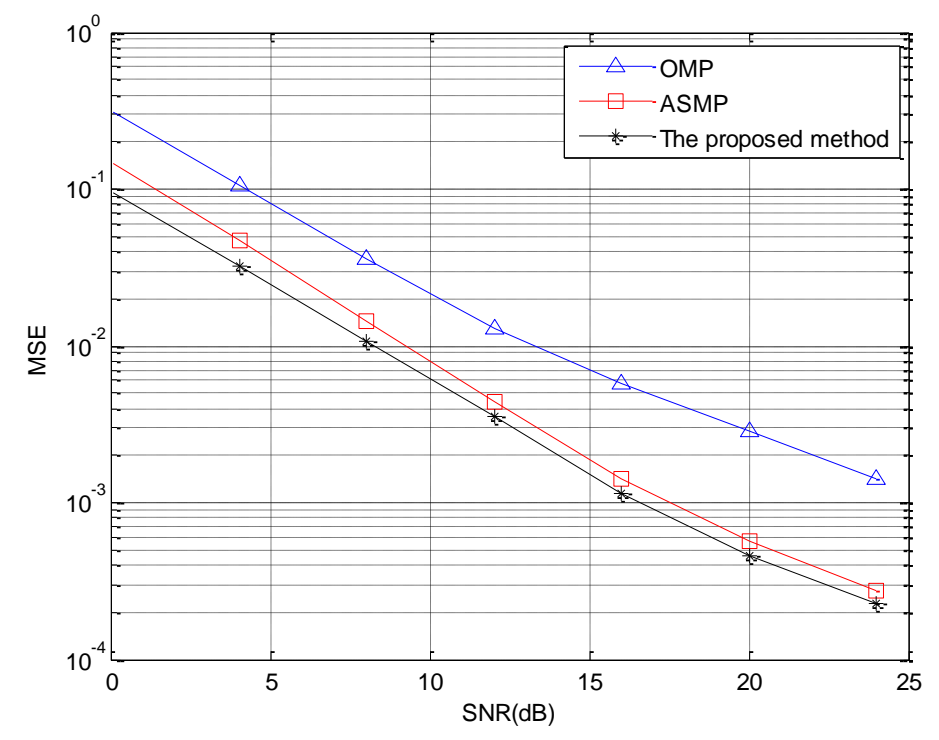

Figure 1. Comparison of MSE Performances of the Channel Estimators

Figure 2 shows the bit-error-rates (BERs) of three channel estimators. And the ASMP based channel estimator has a better performance than OMP based channel estimator. But the proposed method outperforms the other two channel estimators. 
The proposed method can resist noise better than ASMP based channel estimator with about $1.3 \mathrm{~dB}$ gain.

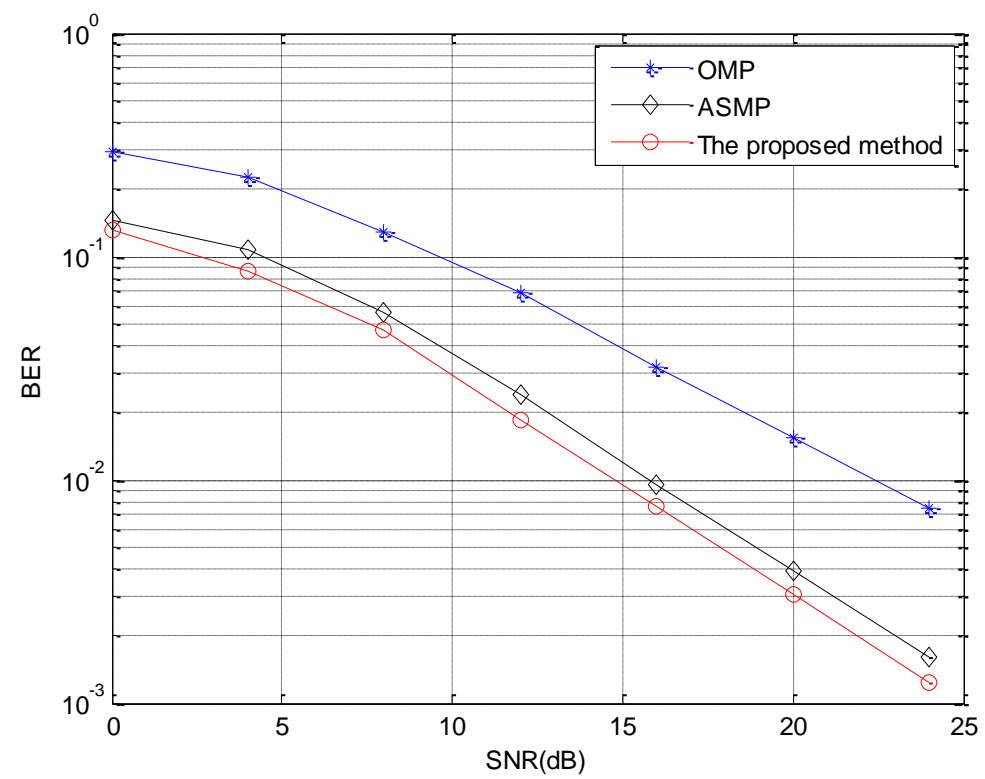

Figure 2. Comparison of BER Performances of the Channel Estimators

\section{Conclusion}

In this paper, we have studied the CS based channel estimation. We modified the ASMP based channel estimation using SVD. The proposed channel estimation does not require the information of channel sparity. Moreover, it has a better performance of resisting noise. The simulation results show that the proposed estimation can resist AWGN than ASMP based channel estimation.

\section{References}

[1] O. Edfors, M. Sandell, J. J. van de Beek, S. K. Wilson and P. O. Böjesson, "OFDM channel estimation by singular value decomposition”, IEEE Trans. Commun., vol. 46, no. 7, (1998), pp. 931-939.

[2] B. Yang, K. B. Letaief, R. S. Cheng and Z. Cao, "Channel estimation for OFDM transmission in multipath fading channels based on parametric channel modeling", IEEE Trans. Commun., vol. 49, no. 3, (2001), pp. 467-479.

[3] Coleri S, Ergen M and Puri A, "Channel estimation techniques based on pilot arrangement in OFDM systems”, IEEE Transactions on Broadcasting, vol. 48, no. 3, (2002), pp.223-229.

[4] J. A. Tropp and A. C. Gilbert, "Signal Recovery From Random Measurements Via Orthogonal Matching Pursuit", IEEE Trans. on Information Theory, vol. 53, no. 12, (2007), pp. 4655-4666..

[5] D. Needell and R. Vershynin, "Uniform Uncertainty Principle and signal recovery via Regularized Orthogonal Matching Pursuit”, Foundations of Computational Math., vol. 9, no. 3, (2009), pp. 317-334.

[6] D. Needell and J. A. Tropp, "CoSaMP: Iterative signal recovery from incomplete and inaccurate samples", Appl. Comput. Harm. Anal., vol. 26, no. 3, (2009), pp. 301-321.

[7] N. Wang, Z. Zhang and G. Gui, "Improved sparse channel estimation for multicarrier systems with compressive sensing", IEEE 14th International Symposium on Wireless Personal Multimedia Communications (WPMC), (2011), pp.1-5.

[8] H. Wang, J. Huang, C. He and Q. Zhang, "An efficient sparse channel estimation method with predetermined sparsity”, IEEE Region 10 Conference (TENCON 2013), (2013), pp. 1-5.

[9] Z. Dong, L. Ge and K. Gong, "Joint Sparsity and Sparse Channel Estimation Algorithm Based on CoSaMP”, Journal of Sichuan University, vol. 46, no. 1, (2014), pp.121-127.

[10] Y. Wu, X. Zhou, W. Chen and X. Liao, "Adaptive sparse channel estimator for OFDM systems", IEEE 2013 International Conference on Wireless Communications \& Signal Processing (WCSP) International Conference, (2013), pp.1-5. 
[11] H. Wu and S.Wang, "Adaptive Sparsity Matching Pursuit Algorithm for Sparse Reconstruction”, IEEE Signal Processing Letters, vol. 19, no. 8, (2012), pp. 471-474.

[12] Y. Peng, Y. He and B.Lin, "Noise signal recovery algorithm based on singular value decomposition in compressed sensing", Chinese Journal of Scientific Instrument, vol. 33, no. 12, (2012), pp. 2655-2660.

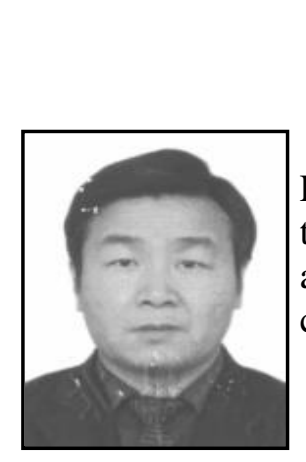

\section{Authors}

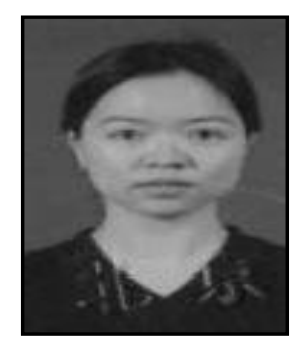

Liu Zhiyong, he received the B.E. degree from Beijing Institute of Light Industry in 1988, senior engineer. He has long been engaged in the communication industry and has a deep understanding and awareness in wireless communication. His research area is wireless communications, Smart grid, and Electric power information system.

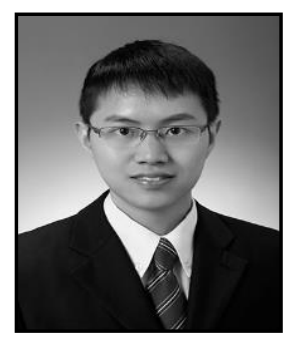

Liu Weicai, he received the B.S degree in Department of Applied Physics, Beihang University, Beijing, China, in 2010. He is now pursuing the M.S. degree in School of Electronic Engineering, Beijing University of Posts and Telecommunications (BUPT), Beijing, China. His research area is wireless communications. 